\title{
Selection of vasodilator therapy for severe Raynaud's phenomenon by sequential arterial infusion
}

\author{
I JON RUSSELL AND RICHARD A WALSH \\ From the Department of Medicine, Divisions of Clinical Immunology and Cardiology, the University of \\ Texas Health Science Center at San Antonio, USA.
}

SUMMARY Two young adults with impending gangrene due to persistent Raynaud's vasospasm were studied by sequential intra-arterial (IA) infusion of three vasodilatory drugs while arterial pressure and plethysmographic digital pulse volume were continuously monitored. The results of the IA infusion accurately predicted relief from vasospastic symptoms by oral drugs of the same class. This method may be useful in selecting the most effective vasodilator for patients with severe vasospasm.

Key words: vasodilatation, tolazoline, hydralazine, diltiazem, phenoxybenzamine.

Raynaud's phenomenon may occur as an isolated problem or as one manifestation of a major clinical syndrome. The management of infrequent vasospastic episodes can be as simple as avoidance of cold, wearing of gloves, or windmilling the affected arm to override the increased vascular tone. ${ }^{1}$ For patients with very frequent or persistent attacks a variety of chemotherapeutic remedies have been used. $^{2-4}$ It has previously been difficult to predict which vasodilator will be most effective for any individual patient. Occasionally that information is needed urgently to save digits from permanent injury. The following case studies illustrate a method of sequential intra-arterial infusions of vasoactive drugs, monitored by digital plethysmography, which identified effective therapy for two patients with severe Raynaud's phenomenon.

\section{Materials and methods}

Two patients with impending digital gangrene were taken to a fully equipped cardiac catheterisation laboratory. An arterial cannula was placed in the left brachial artery with a three-way stopcock to allow infusion of drug and monitoring of the arterial pressure. Measurements of digital pulse amplitude and finger systolic pressure were obtained on the left third finger by means of a Pulse Volume Recorder

Accepted for publication 3 September 1984

Correspondence to I Jon Russell, MD, Department of Medicine, University of Texas Health Science Center, 7703 Floyd Curl Drive, San Antonio, TX, 78284, USA.
(PVR-4, Life Sciences Incorporated, Greenwich, CT). This semisegmental plethysmograph uses an air-containing cuff round the proximal or distal phalanx to plot pulsations as wave forms on a strip recorder. ${ }^{5}$ By inflating a second cuff round the proximal phalanx the pressure required to obliterate pulsation at the distal phalanx was determined, and it was defined as the finger systolic pressure.

After baseline measurements were taken, medications were administered sequentially with continuous monitoring of digital pulse volume and central pressures. The drugs used included tolazoline (Priscoline) (5 mg, an alpha adrenergic blocker), diltiazem $(0.64 \mathrm{mg}$, a calcium channel blocker), and hydralazine (5-10 $\mathrm{mg}$, a direct acting arterial dilator). After each infusion tracings were recorded for at least 15 minutes to detect any influence on pulsatile blood flow. A subsequent drug was not given until all effect from the previous drug had resolved. If a vasodilatory drug proved beneficial when given intra-arterially, an oral preparation from the same class was later titrated up to optimal dosage for long term therapy.

\section{Case reports}

CASE 1

A 28-year-old Hispanic female smoker presented with a 13-year history of progressive systemic sclerosis (PSS) and severe Raynaud's phenomenon. Previous evaluation of her PSS revealed oesophageal hypomotility, restrictive lung disease, 
and cor pulmonale, but her renal function had remained normal. Treatment of her progressive vasospastic ischaemia with alpha-methyldopa, prazosin, intra-arterial reserpine, and nitroprusside had been unsuccessful.

In February 1983 she was admitted to hospital because of severe pain in her hands and feet and impending digital gangrene. Examination showed sclerodactyly, digital tuft scars from previous ischaemia, and tightness of her facial skin, but there were no telangiectasis or subcutaneous calcifications. Her peripheral blood and platelet counts were normal. Her blood pressure was $90 / 85 \mathrm{mmHg}$ and the pulse was regular at $84 /$ minute. Pulmonary hypertension and mitral regurgitation were noted. The radial pulses were decreased, while the dorsalis pedis and posterior tibial pulses were not palpable. Her hands and feet were cold and extremely tender to touch, with cyanosis distal to both mid forearms and calves. Ischaemic demarcation was present at the proximal interphalangeal joint of the right index finger and midway on the right great toe. Therefore surgical amputation of several fingers and toes was considered.

Baseline digital plethysmography detected no pulsatile flow as illustrated for the left third finger (Fig. 1). Sequential infusion of tolazoline and diltiazem resulted in falls of $6-10 \mathrm{mmHg}$ in central systolic pressure, but no measurable increase in pulsatile flow was noted. After infusion of hydralazine $5 \mathrm{mg}$ the central arterial pressure increased slightly, with widening of the pulse pressure. Low amplitude pulse waves, already observed at 3 minutes, increased progressively over 20 minutes, while the finger systolic pressure rose from unmeasurable to $72 \mathrm{mmHg}$.

Oral hydralazine was then started at $10 \mathrm{mg}$ every six hours with continuous monitoring. Her blood pressure increased to $120 / 80 \mathrm{mmHg}$, while the digital pulse amplitude continued to rise (Fig. 2). As there was a substantial decrease in digital pain within the first day, her narcotics were discontinued. By the second day both hands and feet were pink and warm. No evidence of orthostasis was seen after three weeks in hospital as her dosage was gradually titrated up to $50 \mathrm{mg}$ of hydralazine every six hours.

\section{CASE 2}

A 27-year-old Hispanic male smoker with no apparent underlying connective tissue disease presented complaining of bilateral hand pain for nine months. He had previously noted typical three-phase colour changes in his hands on exposure to cold, but for one month his fingers had been persistently cyanotic. He wrapped cloth round both hands and wore mittens. Despite being urged to stop smoking he had

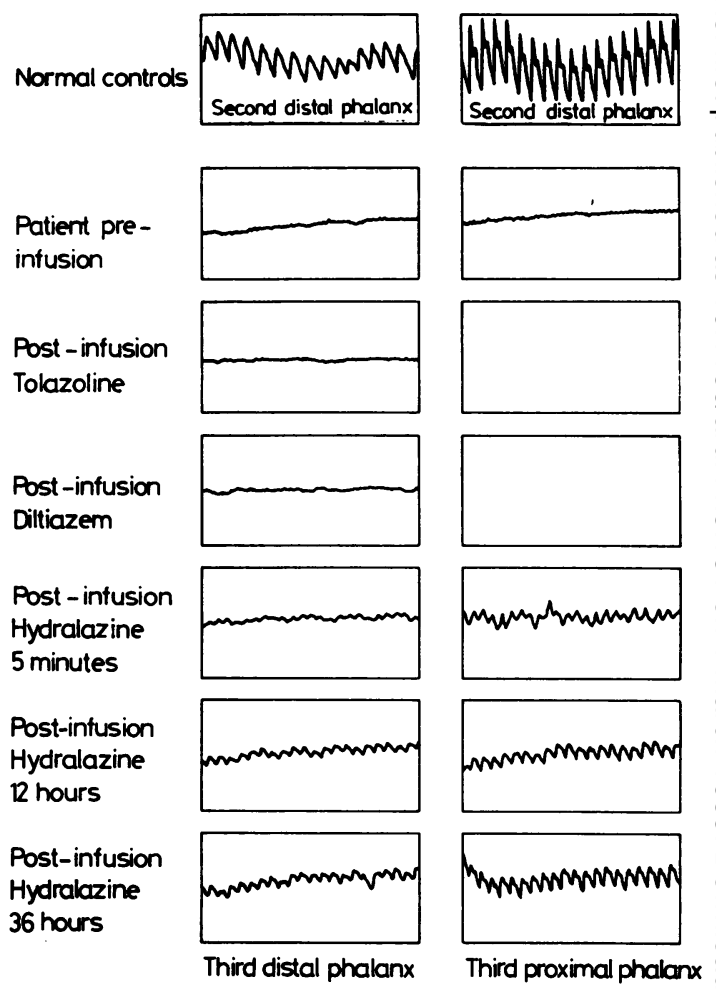

Fig. 1 Digital pulse volume recordings from case 1 . The top two tracings were taken on normal employees at the time of the patient's infusion study. Both represent flow in the distal phalanx of the second finger. The remainder of the tracings are of pulsatile flow in the patient's third distal (left column) and proximal (right column) phalanges. Prior to drug infusion there was no measurable blood flow.

Administration of tolazoline and diltiazem had no effect, but pulsatile flow was apparent three minutes after hydrazaline infusion. This effect was maintained with oral hydrazaline.

been unable to do so. Physical examination revealed cool cyanosis beyond both wrists. Small ulcerations $N$ were noted at the tips of fingers 2,3 , and 4 of both $\tilde{\omega}$ hands, with purulent drainage coming from the second and third fingers on the right. His lowero extremities were unaffected, and peripheral pulses were generally normal except that Allen's test ${ }^{\text {? }}$ disclosed absence of right ulnar arterial flow.

Prior to infusion there was no pulsatile flow in the digits (Fig. 3). Within 1.5 minutes after intra-arterialo administration of tolazoline a nearly normal pulsa- $\frac{\mathbb{Q}}{2}$ tile flow was achieved. His left hand became pinko and throbbing, while the right hand remained 


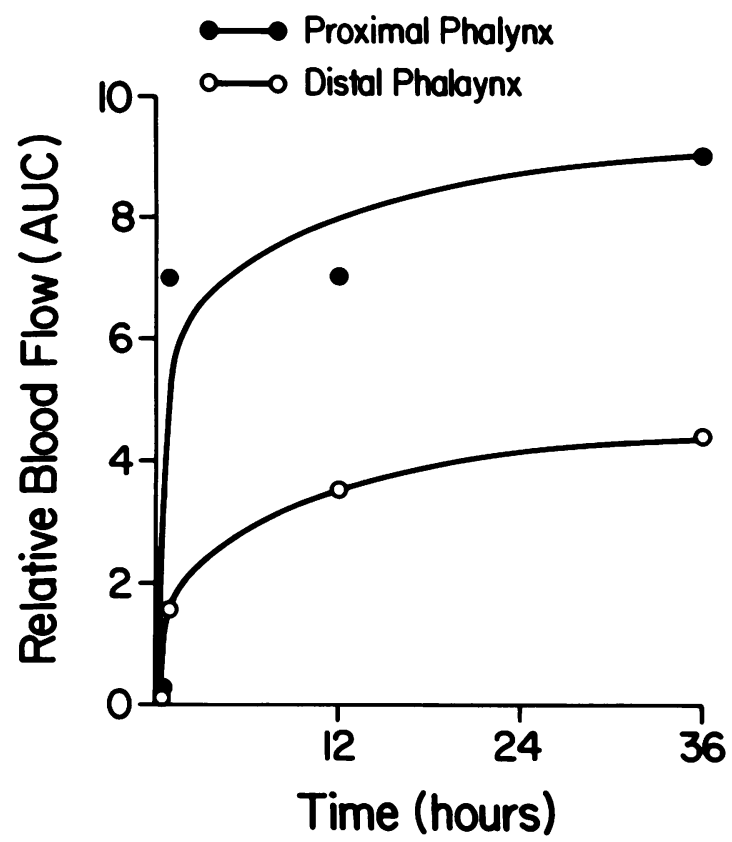

Fig. 2 Relative pulsatile blood flow defined by the area under the curves (AUC) of pulse volume tracings from the left third finger during administration of hydralazine to case 1. Separate curves represent values from the proximal and distal phalanges.

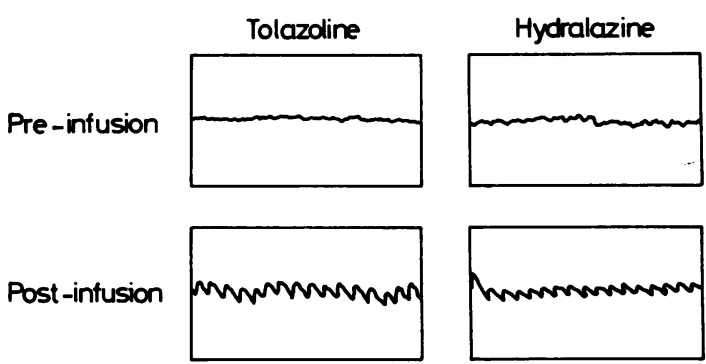

Fig. 3 Digital pulse volume recordings from case 2. The upper left-hand tracing shows no pulsatile flow to the third distal phalanx before administration of tolazoline. Within 90 seconds after tolazoline infusion, pulsatile flow was apparent (lower left-hand tracing). The tracings on the right were taken before and 26 minutes after infusion of hydralazine.

dusky. His blood pressure dropped from $157 / 90$ to $137 / 85 \mathrm{mmHg}$. The effect lasted approximately 15 minutes, after which pulsatile flow had ceased and the duskiness of the left hand returned. Five minutes after hydralazine infusion $(10 \mathrm{mg})$ an increase in pulsatile flow was observed, which persisted for more than 30 minutes with a stable blood pressure at $120 / 90 \mathrm{mmHg}$.

He was initially treated with $10 \mathrm{mg}$ of oral phenoxybenzamine hydrochloride every eight hours, which resulted in plethysmographically observed improvement in blood flow to his hands. Over a period of one month his hand pain resolved, the ulcerations healed despite his continued use of tobacco, and he returned to work. Four months later a return of pain in his fingers and decreased digital blood flow were presumed to be due to phenoxybenzamine tachyphylaxis. The skin had remained intact, but the third fingers of both hands were very tender. The addition of hydralazine, 25 $\mathrm{mg}$ twice daily, provided relief.

\section{Discussion}

The procedure used to select a vasodilator drug for these patients was invasive but seemed warranted when persistent ischaemia endangered viable tissue and prevented healing of ulcers. The convenience of simultaneous plethysmography for detecting changes in blood flow makes this a method by which potentially active agents can be tested. It may also provide the setting in which combinations of individually useful agents could be screened simultaneously for additive or synergistic benefit.

Several logical principles govern the usefulness of this invasive approach. (1) The patient must have persistent vasospasm, or there must be a reproducible method of inducing it without disturbing the monitoring system. (2) Representative agents should be selected from each of several drug classes with different mechanisms of action. (3) The sequence of administration should utilise the more rapidly metabolised drugs first. (4) The dosage of each agent should be sufficient to induce at least a transient change in blood pressure. (5) The instruments used to follow changes in digital blood flow should be capable of making continuous measurements. (6) Subsequent oral administration of the selected drug must be monitored closely.

Hydralazine has apparently not been formally studied for treatment of Raynaud's phenomenon. It has been found beneficial in the management of pulmonary hypertension, ${ }^{67}$ which is also thought to have a vasospastic component. ${ }^{8}$ Indeed its effect on pulmonary vascular resistance and cardiac output ${ }^{9}$ may have contributed to improved digital blood flow. Four additional outpatients with Raynaud's phenomenon in association with normal blood pressure have subsequently been treated with oral hydralazine at doses ranging from 40 to $80 \mathrm{mg} /$ day without a prior invasive study. Each patient improved despite a prior lack of responsiveness to 


\section{Russell, Walsh}

more conventional drugs. Together these observations suggest a need for further study of hydralazine in the treatment of Raynaud's phenomenon.

The authors acknowledge the secretarial assistance of Claire Hammerick.

\section{References}

1 McIntyre D R. A maneuver to reverse Raynaud's phenomenon of the fingers. JAMA 1978; 240: 2760 .

2 Halperin J L, Coffman J D. Pathophysiology of Raynaud's disease. Arch Intern Med 1979; 139: 82-92.
3 Smith C D, McKendry R J R. Controlled trial of nifedipine in the treatment of Raynaud's phenomenon. Lancet 1982; ii 1299-301.

4 Siebold J R, Jageneau A H M. Ketanserin in the treatment of Raynaud's phenomenon (RP). Arthritis Rheum 1983; 26: S27.

5 Gifford R W Jr. The arteriospastic diseases: clinical significance으 and management; peripheral vascular disease. In: Brest A $\mathrm{N}, \overline{\bar{\omega}}$ ed. Cardiovascular clinics. Philadelphia: Davis, 1971.

$6 \mathrm{McWeeney} \mathrm{J,} \mathrm{Finnegan} \mathrm{P.} \mathrm{Vasodilator} \mathrm{therapy} \mathrm{of} \mathrm{pulmonary}$ hypertension in the CRST syndrome. Ir J Med Sci 1982; 151 is $151-4$

7 Rubin L J, Peter R H. Oral hydralazine therapy for primary $\overrightarrow{0}$ pulmonary hypertension. $N$ Engl $J$ Med 1980; 302: 69-73

8 Fahey P J, Utell M J, Condemi J J, Green R M, Hyde R W. $\vec{\omega}$ Raynaud's phenomenon of the pulmonary vasculature. Ches? 1980; 78: $515 \mathrm{~A}$.

9 Koch-Weser J. Hydralazine. N Engl J Med 1976; 295: 320-3. 